\title{
Erratum: Masses, mixing angles and phases of general Majorana neutrino mass matrix
}

\author{
Biswajit Adhikary, ${ }^{a}$ Mainak Chakraborty ${ }^{b}$ and Ambar Ghosal ${ }^{b}$ \\ ${ }^{a}$ Department of Physics, Gurudas College, \\ Narkeldanga, Kolkata-700054, India \\ ${ }^{b}$ Saha Institute of Nuclear Physics, \\ 1/AF Bidhannagar, Kolkata 700064, India \\ E-mail: biswajitadhikary@gmail.com, mainak.chakraborty@saha.ac.in, \\ ambar.ghosal@saha.ac.in
}

ERRATUM TO: JHEP10(2013)043

ARXIV EPRINT: 1307.0988

1. Eq. (3.11) should read as

$$
\cos \left(\beta_{M}-\alpha_{M}\right)=\frac{\left|\left(m_{\nu}\right){ }_{11}\right|^{2}-c_{12}^{4} m_{1}^{2}-s_{12}^{4} m_{2}^{2}}{2 c_{12}^{2} s_{12}^{2} m_{1} m_{2}} .
$$

(In RHS of the Numerator $m_{1}^{4}$ is replaced by $m_{1}^{2}$ ).

2. In eq. (3.15) the term $D$ should read as

$$
D=m_{1} m_{2}\left[2 Z c_{12}^{2} s_{12}^{2}+Z \sin 2 \theta_{12} \cos 2 \theta_{12} s_{13} e^{i \delta} \frac{t_{23}^{2}-1}{t_{23}}-2 s_{12}^{2} c_{12}^{2}\right]
$$

(In the second term of the RHS a missing factor ' $Z$ ' is inserted).

Open Access. This article is distributed under the terms of the Creative Commons Attribution License (CC-BY 4.0), which permits any use, distribution and reproduction in any medium, provided the original author(s) and source are credited. 